\title{
A Case of Lung Adenocarcinoma with Long-Term Response after Late-Onset Pembrolizumab-Induced Acute Adrenal Insufficiency
}

\author{
Kei Sonehara Kazunari Tateishi Taro Hirabayashi Taisuke Araki \\ Yuichi lkuyama Ryosuke Machida Masayuki Hanaoka
}

First Department of Internal Medicine, Shinshu University School of Medicine, Matsumoto, Japan

\section{Keywords}

Adrenal insufficiency · Immune-related adverse events · Lung adenocarcinoma ·

Pembrolizumab · Prognostic factor

\section{Abstract}

Pembrolizumab is an anti-programmed cell death protein-1 antibody that is mainly used for the treatment of non-small cell lung cancer (NSCLC). Immune-related adverse events can be caused by immune checkpoint inhibitors; however, few case reports evaluate the prognosis of patients with NSCLC with late-onset immune-related adverse events. In this case, a 63-yearold man with stage IVA lung adenocarcinoma received pembrolizumab as first-line therapy and achieved a complete response. The patient developed hypothyroidism and skin toxicity owing to pembrolizumab over the course of treatment; however, the patient continued with pembrolizumab. The patient discontinued pembrolizumab after 20 cycles owing to appetite loss from 14 months after the initiation of pembrolizumab. Two months later, the symptoms worsened and the patient was taken to hospital by an ambulance owing to movement difficulty. The patient was diagnosed with acute adrenal insufficiency by endocrinological examinations. The condition of the patient improved after hydrocortisone treatment. Sixteen months have passed without the readministration of pembrolizumab and no recurrence of lung adenocarcinoma has been observed. Late-onset, severe, and diverse immune-related adverse events may be a favorable prognostic factor associated with survival. 
Sonehara et al.: Complete Response after Pembrolizumab-Induced Acute Adrena Insufficiency

\section{Introduction}

Pembrolizumab is an anti-programmed cell death protein-1 (anti-PD-1) antibody that is mainly used for the treatment of non-small cell lung cancer (NSCLC). The efficacy and safety of pembrolizumab have been reported in several studies [1-3]. Immune-related adverse events (irAEs), such as pneumonitis, skin toxicities, colitis, thyroid dysfunction, and hepatitis, are caused by immune checkpoint inhibitors (ICIs) and occur in various parts of the body [4]. Adrenal insufficiency is an uncommon irAE and is reported to occur in $0.5 \%$ of patients with NSCLC who are treated with anti-PD-1 antibodies [5]. The symptoms of adrenal insufficiency are nonspecific, such as appetite loss, nausea, fever, and apathy [6]. A delay in diagnosis may result in the development of severe symptoms. IrAEs may be a useful favorable prognostic factor associated with survival in patients with NSCLC who are treated with ICIs [7]. Additionally, Hosoya et al. [8] reported that the early development of irAEs in patients who received an ICI is associated with a better clinical outcome.

However, few case reports have evaluated the prognosis of patients with NSCLC with late-onset irAEs. Here, we report on a patient with advanced lung adenocarcinoma who maintained a complete response (CR) after late-onset pembrolizumab-induced acute adrenal insufficiency.

\section{Case Presentation}

A 63-year-old man was diagnosed with clinical T1cN3M1b stage IVA lung adenocarcinoma (according to the 8th edition of the TNM Classification of Malignant Tumors). There was a metastatic lesion on the left adrenal gland. After examination of the biopsy tissue, epidermal growth factor receptor mutations, anaplastic lymphoma kinase translocations, and c-ros oncogene 1 translocations were not present, and the programmed cell death protein-ligand 1 (PD-L1) tumor proportion score was 100\%. Thus, pembrolizumab monotherapy was used as first-line treatment.

After 9 cycles of $200 \mathrm{mg}$ of pembrolizumab every 3 weeks, the patient achieved a CR (Fig. 1). After 15 cycles of pembrolizumab (from day 319 after the initiation of pembrolizumab), the patient developed skin toxicity (grade 2), but the rash improved after treatment with a steroidal anti-inflammatory agent. After 17 cycles of pembrolizumab (day 369), the patient developed hypothyroidism (grade 2) and commenced $25 \mu \mathrm{g}$ of levothyroxine daily. The thyroid-stimulating hormone level was $17.98 \mu \mathrm{IU} / \mathrm{mL}$, the free $\mathrm{T}_{3}$ level was $2.98 \mathrm{pg} / \mathrm{mL}$, and the free $\mathrm{T}_{4}$ level was $0.61 \mathrm{ng} / \mathrm{dL}$. On day 434 , the dose of levothyroxine was increased to $100 \mu \mathrm{g}$ daily, adjusting for the thyroid-stimulating hormone level.

After 20 cycles of pembrolizumab (day 434), anorexia occurred and did not improve. Therefore, pembrolizumab was discontinued. However, the condition of the patient did not improve and the patient gradually became malaise. On day 441, no abnormality was observed in the pituitary gland using magnetic resonance imaging.

On day 484, the patient was taken to the hospital in an ambulance owing to movement difficulty. Physical examination upon admission revealed consciousness at E4V5M6, a body temperature of $39.1^{\circ} \mathrm{C}$, blood pressure of $126 / 76 \mathrm{~mm} \mathrm{Hg}$, regular pulse of 120 beats/min, respiratory rate of 12 breaths/min, and arterial blood oxygen saturation of $93 \%$ (nasal $1 \mathrm{~L} /$ min). The body weight was $11.2 \mathrm{~kg}$ lower than that at the initiation of pembrolizumab (from 70 to $58.8 \mathrm{~kg}$ ). The laboratory findings are shown in Table 1 . A blood analysis revealed an elevated inflammatory response: white blood cells at 13,220/ $\mu \mathrm{L}, \mathrm{C}$-reactive protein at 9.97 $\mathrm{mg} / \mathrm{dL}$, hyponatremia (sodium concentration: $132 \mathrm{mEq} / \mathrm{L}$ ), hypoglycemia (sugar concentration: $48 \mathrm{mg} / \mathrm{dL}$ ), an elevated creatinine (Cre) level $(1.11 \mathrm{mg} / \mathrm{dL})$, and an elevated creat-

\section{Karger'}




\section{Case Reports in Oncology}

\begin{tabular}{|c|c|}
\hline ase Rep Oncol 2021;14:1-7 & \\
\hline DOI: $10.1159 / 000508068$ & $\begin{array}{l}\text { (c) } 2021 \text { The Author(s). Published by S. Karger AG, Basel } \\
\text { www.karger.com/cro }\end{array}$ \\
\hline
\end{tabular}

Sonehara et al.: Complete Response after Pembrolizumab-Induced Acute Adrenal Insufficiency

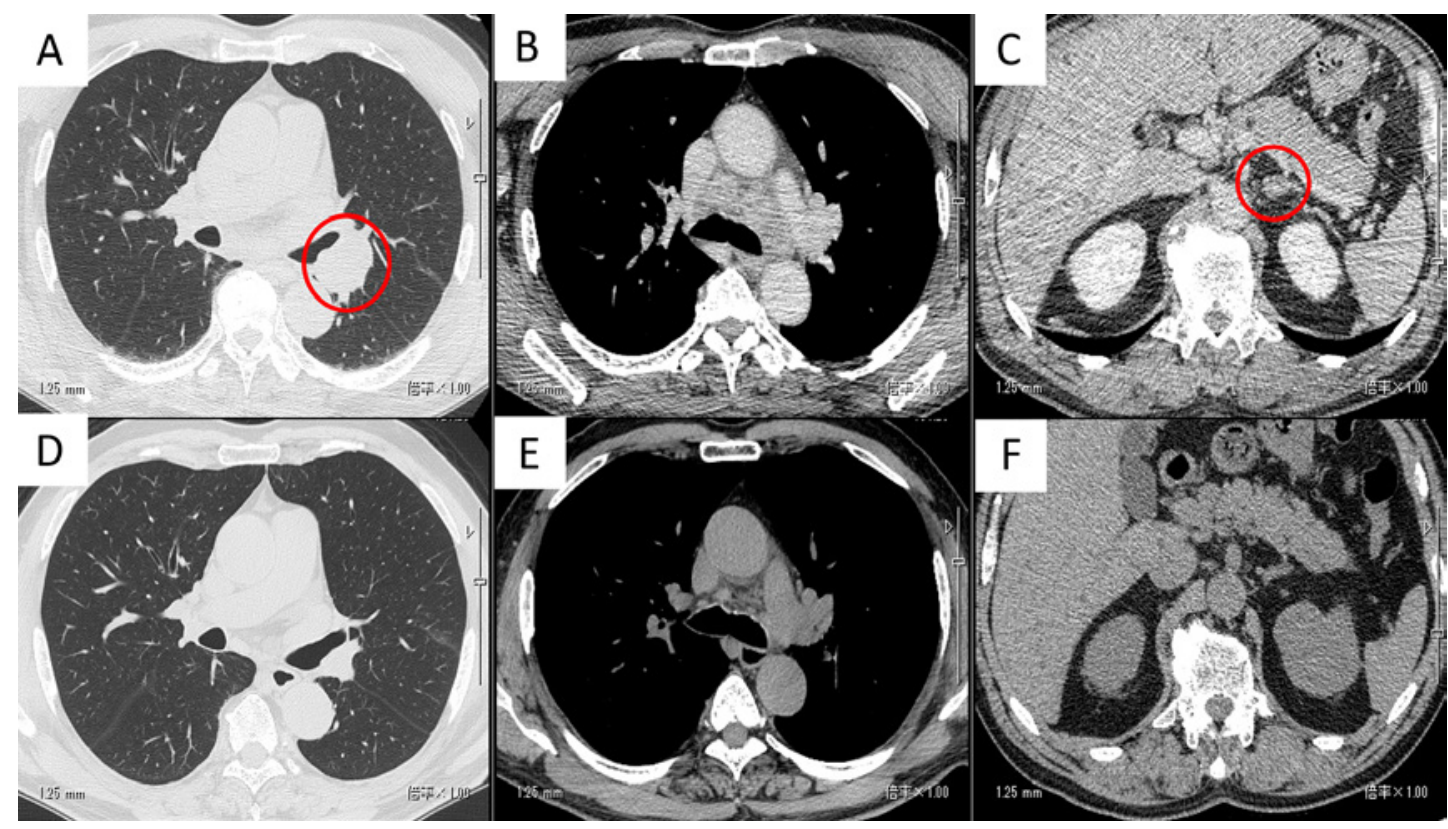

Fig. 1. Chest computed tomography scans. A-C Performed before the initiation of pembrolizumab. D-F Performed on day 196 (after 9 cycles of pembrolizumab) after the initiation of pembrolizumab. A A 2.2-cm tumor in the lower lobe of the left lung was seen (red circle). The primary lesion was observed. B Mediastinal lymph node metastasis was observed. C Left adrenal metastasis was observed. D, E The size of the primary lesion and mediastinal lymph node was reduced. F The left adrenal metastasis had disappeared.

Table 1. Laboratory findings

\begin{tabular}{|c|c|c|c|}
\hline Hematology & & Chloride & $99 \mathrm{mEq} / \mathrm{l}$ \\
\hline White blood cells & $13,220 / \mu \mathrm{L}$ & Calcium & $9.7 \mathrm{mg} / \mathrm{dL}$ \\
\hline Neutrophils & $63.3 \%$ & C-reactive protein & $9.97 \mathrm{mg} / \mathrm{dL}$ \\
\hline Lymphocytes & $21.8 \%$ & Glucose & $48 \mathrm{mg} / \mathrm{dL}$ \\
\hline Monocytes & $12.0 \%$ & Procalcitonin & $3.83 \mathrm{ng} / \mathrm{dL}$ \\
\hline Eosinophils & $2.3 \%$ & \multicolumn{2}{|c|}{ Arterial blood gas (nasal 1 L/min) } \\
\hline Basophils & $0.6 \%$ & $\mathrm{pH}$ & 7.493 \\
\hline Red blood cells & $387 \times 10^{4} / \mu \mathrm{L}$ & $\mathrm{PaO}_{2}$ & 111 Torr \\
\hline Hemoglobin & $11.8 \mathrm{~g} / \mathrm{dL}$ & $\mathrm{PaCO}_{2}$ & 28.4 Torr \\
\hline Hematocrit & $34.6 \%$ & $\mathrm{CO}_{3}^{-}$ & $21.5 \mathrm{mmol} / \mathrm{L}$ \\
\hline Platelets & $8.7 \times 10^{4} / \mu \mathrm{L}$ & Urinalysis & \\
\hline Blood chemistry & & Gravity & 1.020 \\
\hline Total protein & $6.9 \mathrm{~g} / \mathrm{dL}$ & Protein & $(-)$ \\
\hline Albumin & $3.9 \mathrm{~g} / \mathrm{dL}$ & Occult blood & $(-)$ \\
\hline Urea nitrogen & $21.3 \mathrm{mg} / \mathrm{dL}$ & Endocrinology & \\
\hline Creatinine & $1.11 \mathrm{mg} / \mathrm{dL}$ & Thyroid-stimulating hormone & $20.35 \mu \mathrm{IU} / \mathrm{mL}(0.5-5.0)$ \\
\hline Uric acid & $5.3 \mathrm{mg} / \mathrm{dL}$ & Free triiodothyronine & $3.02 \mathrm{pg} / \mathrm{mL}(2.3-4.0)$ \\
\hline Aspartate aminotransferase & $50 \mathrm{U} / \mathrm{L}$ & Free thyroxine & $1.46 \mathrm{ng} / \mathrm{dL}(0.9-1.7)$ \\
\hline Lactate dehydrogenase & $291 \mathrm{U} / \mathrm{L}$ & Adrenocorticotropic hormone & $1.1 \mathrm{pg} / \mathrm{mL}(7.2-63.3)$ \\
\hline Creatinine kinase & $440 \mathrm{U} / \mathrm{L}$ & Cortisol & $3.8 \mu \mathrm{g} / \mathrm{dL}(5.0-15.0)$ \\
\hline Sodium & $132 \mathrm{mEq} / \mathrm{L}$ & & \\
\hline Potassium & $6 \mathrm{mEq} / \mathrm{L}$ & & \\
\hline
\end{tabular}




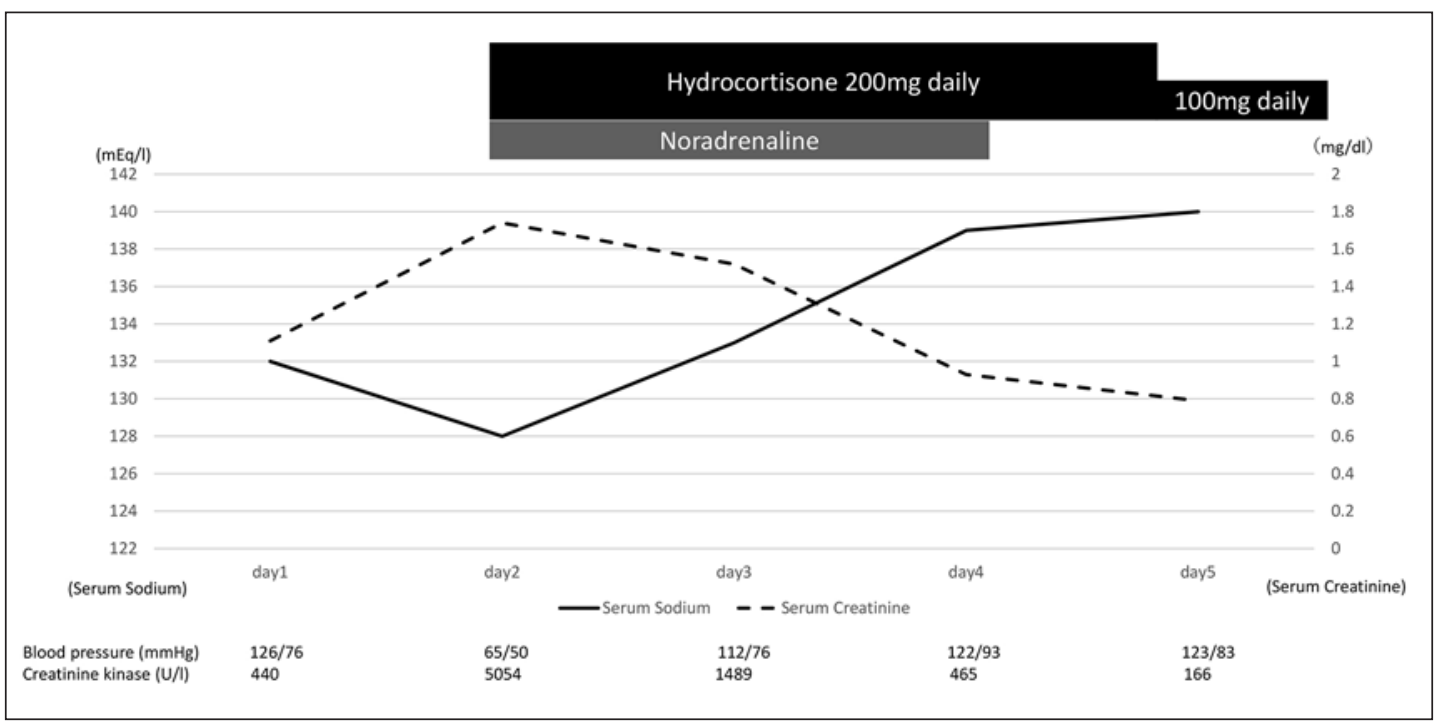

Fig. 2. Clinical course from day 1 to day 5 after admission.

inine kinase (CK) level (440 U/L). A chest X-ray did not reveal any abnormalities. Therefore, the patient was diagnosed with dehydration owing to some infectious disease and fluid replacement and antibiotic treatment was commenced on hospitalization.

On day 2 after admission, a blood analysis revealed that the Cre level $(1.74 \mathrm{mg} / \mathrm{dL}), \mathrm{CK}$ level (5,054 U/L), and hyponatremia (sodium concentration: $128 \mathrm{mEq} / \mathrm{L}$ ) were further exacerbated. Additionally, blood pressure had lowered to $65 / 50 \mathrm{~mm} \mathrm{Hg}$. On endocrine examination, low levels of cortisol and adrenocorticotropic hormone were revealed $(3.8 \mu \mathrm{g} / \mathrm{dL}$ and $1.1 \mathrm{pg} / \mathrm{mL}$, respectively). The patient was diagnosed with acute adrenal insufficiency (grade 4) with prerenal failure induced by pembrolizumab. Hydrocortisone (200 mg daily) and noradrenaline were added to the treatment. The clinical course is shown in Figure 2. On day 5 after admission, the patient experienced an improvement in appetite. Additionally, serum sodium, Cre, and CK levels had improved to within the normal range. On day 8 after admission, the dose of hydrocortisone was reduced to $30 \mathrm{mg}$ daily (orally). The patient was discharged on day 21 after admission because there was no relapse of symptoms after a reduction of oral hydrocortisone to $20 \mathrm{mg}$ daily.

With regard to the subsequent clinical course of the patient, pembrolizumab has been discontinued and the administration of oral hydrocortisone (reduced to $15 \mathrm{mg}$ daily on day 733) has continued without relapse of adrenal insufficiency. On day 984, a chest computed tomography scan showed no recurrence of lung adenocarcinoma, and the patient is being monitored without treatment (Fig. 3).

\section{Discussion}

Adrenal insufficiency is a rare adverse event induced by anti-PD-1 antibodies [9]. A previous study reported that the median time for the development of adrenal insufficiency is 5.9 months (range 3.2-12.6) and 4 of 10 patients experience another irAE [10]. The latestonset adrenal insufficiency in a previous case report occurred at 15 months [11]. With regard to the treatment of adrenal insufficiency, corticosteroid replacement therapy is useful and should be administered immediately. 
A

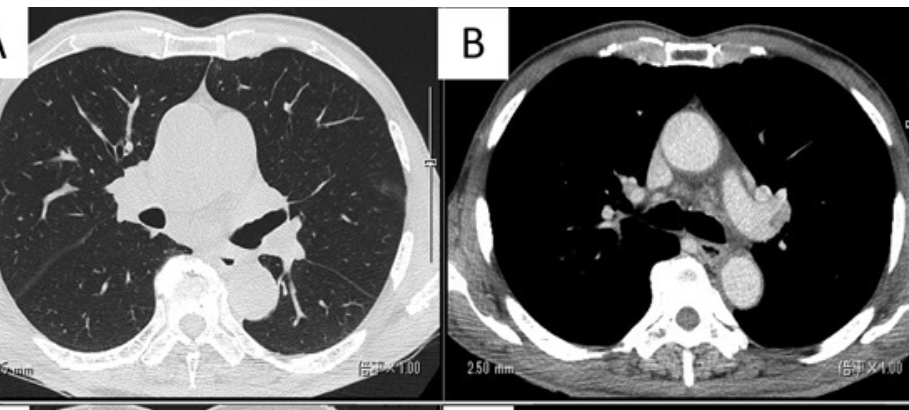

Sonehara et al.: Complete Response after Pembrolizumab-Induced Acute Adrenal Insufficiency

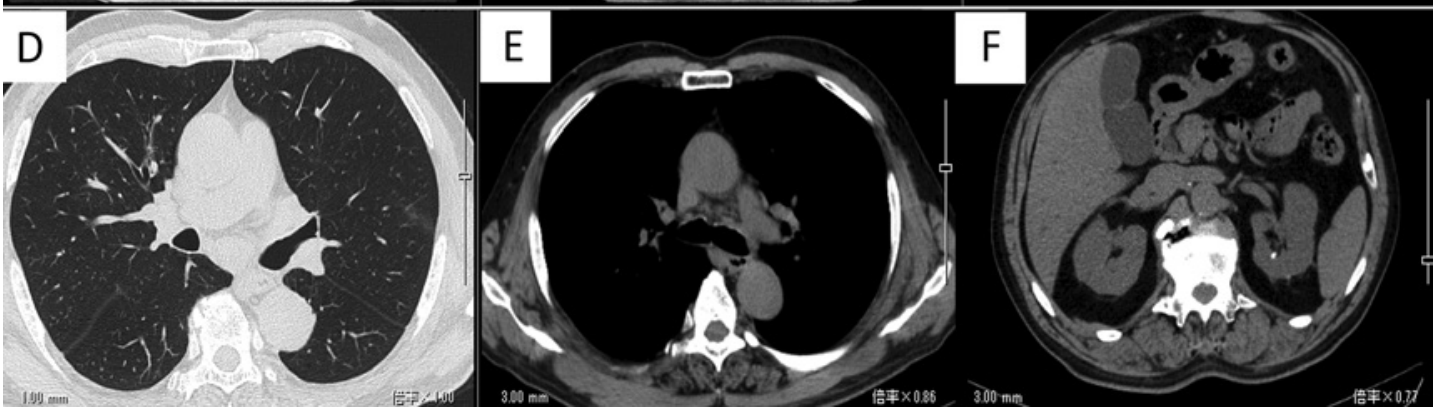

Fig. 3. Chest computed tomography scans. A-C Performed on day 468 after the initiation of pembrolizumab. D-F Performed on day 964 after the initiation of pembrolizumab. There was no recurrence of lung adenocarcinoma, and the patient maintained a complete response.

In our case, as the patient had experienced appetite loss 2 months before the onset of adrenal insufficiency, adrenal insufficiency might have developed 14 months after the initiation of pembrolizumab. Thus, earlier administration of hydrocortisone might have avoided the acute adrenal insufficiency. It is unclear whether the incidence of adrenal insufficiency increased the risk of the development of other irAEs. However, considering a previous report [10] and that this case involved three other irAEs, other irAEs may be a risk factor for the incidence of adrenal insufficiency. A previous phase I trial of patients treated with anti-PD-1 antibodies indicated that high doses of hydrocortisone $(3-10 \mathrm{mg} / \mathrm{kg}$ ) are used to treat adrenal insufficiency [12]. In our case, the initial dose of hydrocortisone was $200 \mathrm{mg}$ daily (3.4 mg/ $\mathrm{kg}$ ) for adrenal insufficiency (grade 4) and the symptoms improved. One week later, the dose was gradually reduced to $30 \mathrm{mg}$ daily $(0.5 \mathrm{mg} / \mathrm{kg})$. Thus, in the case of mild adrenal insufficiency (grade 1 or grade 2), initial therapeutic glucocorticoid doses can be lower than previously reported [12].

It is unclear whether corticosteroid therapy for irAEs influences prognosis. Several studies have suggested that the incidence of irAEs is a favorable prognostic factor $[7,13]$. Toi et al. [13] reported that the median progression-free survival (PFS) in their "irAE induced by nivolumab monotherapy" group was significantly longer than that in the non-irAE group (12.0 vs. 3.6 months, $p=0.013$ ). Additionally, the development of irAEs is a predictive factor for response to nivolumab (odds ratio: $0.11, p<0.001$ ). Ida et al. [10] reported that the median overall survival (OS) of their patients with adrenal insufficiency was favorable at 15.4 months; thus, it may be possible to continue ICI treatment with a corticosteroid. A previous study reported that the median OS in an ICI interruption group owing to irAEs was significantly shorter than that in an ICI continuation group (8.3 vs. 14.5 months, $p=0.008$ ) [14]. However, a retrospective study reported that the PFS and OS in ICI retreatment cohorts who achieved a partial response or CR before the first onset of irAEs are not significantly longer than those in ICI discontinuation cohorts [15]. It is still unclear whether the onset, diversity, and severity 
of irAEs is associated with PFS and OS, and which cases continue to benefit from the anti-PD-1 antibody without retreatment.

In our case, the patient has not been retreated with pembrolizumab, in consultation with the patient, because a CR was achieved. Patients who have achieved or nearly achieved a CR after anti-PD-1 antibody treatment when a late-onset and severe irAE develops may continue to benefit from the anti-PD-1 antibody without retreatment.

In conclusion, we presented a patient with NSCLC with a long-term response after lateonset pembrolizumab-induced acute adrenal insufficiency. This case suggests that the occurrence of late-onset, severe, and diverse irAEs may be associated with the sustained efficacy of an anti-PD-1 antibody; thus, an irAE may be a favorable prognostic factor associated with survival.

\section{Statement of Ethics}

The study was conducted in accordance with the principles of the Declaration of Helsinki. The authors declare that the subject has given his informed written consent to publish his case (including publication of images).

\section{Conflict of Interest Statement}

The authors have no conflicts of interest to declare.

\section{Funding Sources}

The authors did not receive any funding.

\section{Author Contributions}

K.S. and K.T. wrote the manuscript; K.S., K.T., T.H., T.A., Y.I., R.M., and M.H. collected and analyzed the data; K.T. reviewed the paper.

\section{References}

1 Reck M, Rodríguez-Abreu D, Robinson AG, Hui R, Csőszi T, Fülöp A, et al. Pembrolizumab versus chemotherapy for PD-L1-positive non-small-cell lung cancer. N Engl J Med. 2016 Nov;375(19):1823-33.

2 Herbst RS, Baas P, Kim DW, Felip E, Pérez-Gracia JL, Han JY, et al. Pembrolizumab versus docetaxel for previously treated, PD-L1-positive, advanced non-small-cell lung cancer (KEYNOTE-010): a randomised controlled trial. Lancet. 2016 Apr;387(10027):1540-50.

3 Mok TSK, Wu YL, Kudaba I, Kowalski DM, Cho BC, Turna HZ, et al. Pembrolizumab versus chemotherapy for previously untreated, PD-L1-expressing, locally advanced or metastatic non-small-cell lung cancer (KEYNOTE042): a randomised, open-label, controlled, phase 3 trial. Lancet. 2019 May;393(10183):1819-30.

4 Spain L, Diem S, Larkin J. Management of toxicities of immune checkpoint inhibitors. Cancer Treat Rev. 2016 Mar; 44:51-60.

5 Wang PF, Chen Y, Song SY, Wang TJ, Ji WJ, Li SW, et al. Immune-related adverse events associated with antiPD-1/PD-L1 treatment for malignancies: a meta-analysis. Front Pharmacol. 2017 Oct;8:730.

6 Yanase T, Tajima T, Katabami T, Iwasaki Y, Tanahashi Y, Sugawara A, et al. Diagnosis and treatment of adrenal insufficiency including adrenal crisis: a Japan Endocrine Society clinical practice guideline [Opinion]. Endocr J. 2016 Sep;63(9):765-84. 
7 Akamatsu H, Murakami E, Oyanagi J, Shibaki R, Kaki T, Takase E, et al. Immune-related adverse events by immune checkpoint inhibitors significantly predict durable efficacy even in responders with advanced nonsmall cell lung cancer. Oncologist. 2020;25(4):e679.

8 Hosoya K, Fujimoto D, Morimoto T, Kumagai T, Tamiya A, Taniguchi Y, et al. Association between early immune-related adverse events and clinical outcomes in patients with non-small cell lung cancer treated with immune checkpoint inhibitors. Clin Lung Cancer. 2020;21(4):e315-e328.

9 Byun DJ, Wolchok JD, Rosenberg LM, Girotra M. Cancer immunotherapy - immune checkpoint blockade and associated endocrinopathies. Nat Rev Endocrinol. 2017 Apr;13(4):195-207.

10 Ida H, Goto Y, Sato J, Kanda S, Shinno Y, Morita R, et al. Clinical characteristics of adrenal insufficiency as an immune-related adverse event in non-small-cell lung cancer. Med Oncol. 2020 Mar;37(4):30.

11 Boudjemaa A, Rousseau-Bussac G, Monnet I. Late-onset adrenal insufficiency more than 1 year after stopping pembrolizumab. J Thorac Oncol. 2018 Mar;13(3):e39-40.

12 Corsello SM, Barnabei A, Marchetti P, De Vecchis L, Salvatori R, Torino F. Endocrine side effects induced by immune checkpoint inhibitors. J Clin Endocrinol Metab. 2013 Apr;98(4):1361-75.

13 Toi Y, Sugawara S, Kawashima Y, Aiba T, Kawana S, Saito R, et al. Association of immune-related adverse events with clinical benefit in patients with advanced non-small-cell lung cancer treated with nivolumab. Oncologist. 2018 Nov;23(11):1358-65.

14 Ksienski D, Wai ES, Croteau N, Fiorino L, Brooks E, Poonja Z, et al. Efficacy of nivolumab and pembrolizumab in patients with advanced non-small-cell lung cancer needing treatment interruption because of adverse events: a retrospective multicenter analysis. Clin Lung Cancer. 2019 Jan;20(1):e97-106.

15 Santini FC, Rizvi H, Plodkowski AJ, Ni A, Lacouture ME, Gambarin-Gelwan M, et al. Safety and efficacy of re-treating with immunotherapy after immune-related adverse events in patients with NSCLC. Cancer Immunol Res. 2018 Sep;6(9):1093-9. 